\title{
Immediate effects of EVA midsole resilience and upper shoe structure on running biomechanics: a machine learning approach
}

\author{
Andrea N Onodera ${ }^{1,2}$, Wilson Pires Gavião Neto ${ }^{3}$, Maria Isabel Roveri ${ }^{1}{ }^{,}$Wagner R Oliveira $^{2}$, Isabel CN Sacco \\ Corresp. 1 \\ 1 Physical Therapy, Speech and Occupational Therapy department, University of São Paulo, School of Medicine, Sao Paulo, Sao Paulo, Brazil \\ 2 Dass Nordeste Calçados e Artigos Esportivos Inc, Ivoti, Rio Grande do Sul, Brazil \\ 3 School of Engeneering \& IT, Centro Universitário Ritter dos Reis, Porto Alegre, Rio Grande do Sul, Brazil \\ Corresponding Author: Isabel CN Sacco \\ Email address: icnsacco@usp.br
}

Background. Resilience of midsole material and the upper structure of the shoe are conceptual characteristics that can interfere in running biomechanics patterns. Artificial intelligence techniques can capture features from the entire waveform, adding new perspective for biomechanical analysis. This study tested the influence of shoe midsole resilience and upper structure on running kinematics and kinetics of non-professional runners by using feature selection, information gain, and artificial neural network analysis.

Methods. Twenty-seven experienced male runners $(63 \pm 44 \mathrm{~km} /$ week run) ran in four-shoe design that combined two resilience-cushioning materials (low and high) and two uppers (minimalist and structured). Kinematic data was acquired by 6 infrared cameras at $300 \mathrm{~Hz}$, and ground reaction forces were acquired by 2 force plates at $1200 \mathrm{~Hz}$. We conducted a Machine Learning analysis to identify features from the complete kinematic and kinetic time series and from 42 discrete variables that had better discriminate the four shoes studied. And for that, we built an input data matrix of dimensions 1080 (10 trials $x 4$ shoes $\times 27$ subjects) $\times 1254$ ( 3 joints $\times 3$ planes of movement $\times 101$ data points +3 vectors forces $\times 101$ data points +42 discrete calculated kinetic and kinematic features).

Results. The applied feature selection by information gain and artificial neural networks successfully differentiated the two resilience materials using $200(16 \%)$ biomechanical variables with an accuracy of $84.8 \%$ by detecting alterations of running biomechanics, and the two upper structures with an accuracy of $93.9 \%$.

Discussion. The discrimination of midsole resilience resulted in lower accuracy levels than did the discrimination of the shoe uppers. In both cases, the ground reaction forces were among the 25 most relevant features.The resilience of the cushioning material caused significant effects on initial heel impact, while the effects of different uppers were distributed along the stance phase of running. Biomechanical changes due to shoe midsole resilience seemed to be subject-dependent, while those due to upper structure seemed to be subject-independent. 
1

2

3 Andrea N Onodera ${ }^{1,2}$, Wilson P Gavião Neto ${ }^{3}$, Maria Isabel Roveri ${ }^{1}$, Wagner R Oliveira ${ }^{2}$, Isabel

4 5

$6{ }^{1}$ University of São Paulo, School of Medicine, Physical Therapy, Speech and Occupational

7 Therapy dept., São Paulo, SP, Brazil.

$8 \quad{ }^{2}$ Dass Nordeste Calçados e Artigos Esportivos Inc, Ivoti, Rio Grande do Sul, Brazil.

$9{ }^{3}$ Ritter dos Reis University Center - UNIRITTER - Laureate International Universities, School of 10 Engeneering \& IT and Master of Design, Porto Alegre, RS, Brazil.

11

12

Isabel CN Sacco (Corresponding Author)

13

E-mail: icnsacco@usp.br 


\section{Abstract}

Background. Resilience of midsole material and the upper structure of the shoe are conceptual characteristics that can interfere in running biomechanics patterns. Artificial intelligence techniques can capture features from the entire waveform, adding new perspective for biomechanical analysis. This study tested the influence of shoe midsole resilience and upper structure on running kinematics and kinetics of non-professional runners by using feature selection, information gain, and artificial neural network analysis.

Methods. Twenty-seven experienced male runners $(63 \pm 44 \mathrm{~km} /$ week run) ran in four-shoe design that combined two resilience-cushioning materials (low and high) and two uppers (minimalist and structured). Kinematic data was acquired by 6 infrared cameras at $300 \mathrm{~Hz}$, and ground reaction forces were acquired by 2 force plates at $1200 \mathrm{~Hz}$. We conducted a Machine Learning analysis to identify features from the complete kinematic and kinetic time series and from 42 discrete variables that had better discriminate the four shoes studied. And for that, we built an input data matrix of dimensions 1080 (10 trials $\times 4$ shoes $\times 27$ subjects) $\times 1254$ ( 3 joints x 3 planes of movement $\times 101$ data points +3 vectors forces $\times 101$ data points +42 discrete calculated kinetic and kinematic features).

Results. The applied feature selection by information gain and artificial neural networks successfully differentiated the two resilience materials using $200(16 \%)$ biomechanical variables with an accuracy of $84.8 \%$ by detecting alterations of running biomechanics, and the two upper structures with an accuracy of $93.9 \%$.

Discussion. The discrimination of midsole resilience resulted in lower accuracy levels than did the discrimination of the shoe uppers. In both cases, the ground reaction forces were among 
37 the 25 most relevant features. The resilience of the cushioning material caused significant

38 effects on initial heel impact, while the effects of different uppers were distributed along the

39 stance phase of running. Biomechanical changes due to shoe midsole resilience seemed to be

40 subject-dependent, while those due to upper structure seemed to be subject-independent. 
41

42

\section{Introduction}

Sports shoes have many roles in running; among them, providing adequate impact-force absorption (Clarke et al. 1983; Hennig 2011), stability for foot/ankle movements (Cheung et al. 2011) and comfort (Nigg 2010). These roles have been the most studied in running and shoe biomechanics so far. Running shoes are basically constituted by upper, midsole and sole. Among different possible combinations of these three elements in the shoe construction, the upper is definitely the one more subject to variations in its construction, such as color, model design, added elements and materials, and certainly, the last two factors will have a particular influence on running biomechanics. Runners select a comfortable running shoe using their own comfort criteria (Nigg et al. 2015) and, because the shoe upper maintains a large contact area with the foot, it would have a stronger influence over fit and comfort, which in turn would impact in runner's kinematic and kinetic strategies during practice and competitions. It has been demonstrated that a firmer foot contact with a shoe resulted in lower loading rates due to a better coupling of foot-footwear, which optimizes the use of the midsole impact absorption technology by favoring a better foot positioning inside the shoe (Hagen \& Hennig 2009). Investigate the isolated influence of upper types in running biomechanics would help runners to have a more comprehensive and efficient approach in the shoe construction process, as well in the choice of the running shoe by runners.

9 Nevertheless, the most manipulated and studied shoe part in biomechanics is still the midsole (Maclean et al. 2009; Milani et al. 1997; Nigg et al. 2003; Worobets et al. 2014). The majority of shoe companies invests a large amount of time, effort and money development of damping materials technologies, such as gels, air, and springs for supposedly improving sports 
63 performance. Ethylene-vinyl acetate (EVA) is a copolymer of ethylene and vinyl acetate highly

64 elastic sintered to form a porous material similar to rubber, yet with excellent toughness. Its

65 porous elastomeric characteristic is much more flexible as low-density polyethylene, commonly

66 used in shoes construction, and because of its properties of resistance, flexibility, temperature

67 toughness, it has been one of the most used copolymer in the shoe midsole construction

68 (Verdejo \& Mills 2004; Wang et al. 2012).

69 The midsole hardness is the most explored physical characteristic of the midsole in

biomechanical studies (Clarke et al. 1983; Hennig et al. 1996; Kersting \& Brüggemann 2006;

Maclean et al. 2009; Milani et al. 1997; Nigg et al. 2012). Running with hard shoes resulted in

same peak magnitude vertical GRF as running with soft ones (Clarke et al. 1983; Kersting \&

Brüggemann 2006; Nigg et al. 1987) and faster time to achieve the first peak (Clarke et al.

1983). Therefore, midsole hardness affected the loading rate but not in a proportional rate

(Milani et al. 1997). Most of running kinematic changes due to the midsole hardness occurs at

the ankle joint (Clarke et al. 1983; Hardin et al. 2004; Maclean et al. 2009) and some authors

state that these different midsole hardness lead to different impact perception by runners

(Hennig et al. 1996; Milani et al. 1997), which in turn causes distinct alteration on running

mechanics (Kersting \& Brüggemann 2006; Nigg et al. 2012) and may mislead the real impact damping by shoe midsoles (Hennig et al. 1996; Milani et al. 1997).

Apart from midsole hardness, resilience is also an important mechanical property of midsole that has been seldom studied (Sinclair et al. 2014; Sinclair et al. 2016; Worobets et al. 2014). It represents the energy restored by the cushioning material after an applied force 
85

86

87

88

89

90

91

92

93

adding different kinds of compounds to its formula. EVA added to elastomers could have ideal softness and high resilience characteristics, would have a full-recovery capacity for the next foot step after a heel strike, while a less resilient (more viscous) material would have the capacity of attenuating more energies at initial loading cycles, easily achieving compression flattening after some cycles. It is expected that different resilience materials would mainly reflect different initial impact forces, because more resilient materials will quickly restore the cushioning property while less resilient materials will take a little longer to restore the cushioning property (Sun et al. 2008). Sinclair et al. (2014) have shown that running with shoes with energy return component resulted in greater tibial acceleration peak, calcaneous eversion and internal tibia rotation compared to conventional running shoes. In a later study, they have shown lower oxygen consumption and respiratory exchange ratio with more resilient model of shoes (Sinclair et al. 2016). Worobets et al. (2014) manipulated only the midsole materials, maintaining the upper structure, and also reported lower oxygen consumption when running with a more compliant/resilient midsole condition. However, the isolated effect of resilience changing in shoe midsole is still unknown in running biomechanics.

The majority of biomechanics studies vary the shoe model as a whole to investigate the effects of various structural shoe properties and elements while running (Azevedo et al. 2012; Braunstein et al. 2010; Dixon 2008; McNair \& Marshall 1994). Such an approach deeply interferes with an appropriate differentiation and interpretation of which shoe characteristics most influence the kinetic and kinematic changes during running. The novelty of this study proposal was to manipulate selectively the upper structure and the cushioning material resilience and to investigate the effects of this manipulation in the biomechanics of running. 
107 Identifying more precisely which shoe characteristics really matter for impact attenuation and 108 lower limb kinematic adaptation would help runners to choose more wisely the running shoes 109 regardless the brand or model and direct further running training regimes based on that choice. 110 Nonetheless, individual's mechanical and neuromuscular adaptations to changes in

111 shoes are influenced by mechanical, neurophysiological, anatomical and even psychological 112 factors and, therefore, is likely to observed different individuals using different strategies in 113 response to changes in running shoes (Kersting \& Brüggemann 2006; Nigg et al. 2003). Thereby, 114 one may conclude that regardless the type of shoe modification, the biomechanical responses 115 observed may be subject-dependent.

116 We proposed to identify the relevant biomechanical features which are most affected 117 by different shoes conditions during running, two midsole resiliencies and two upper 118 structures. We adopted an approach based on machine learning (ML), which has been used in 119 the literature to identify crucial features and relevant patterns for classifying and predicting 120 locomotor patterns as being a result of a given health condition (Hoerzer et al. 2015; Muniz et 121 al. 2010b; Schöllhorn et al. 2002), but it has never been used before to identify effects of 122 different running shoes on biomechanics. In contrast to the related literature, our ML strategy 123 is entirely supervised, and it consisted of using Information Gain (IG) to select important 124 features and Artificial Neural Networks (ANN) to classify the different shoe resiliencies and 125 upper types. Our assumptions were: (h1) low versus high resilient cushioning effects on running 126 kinematics and ground reaction forces are classifiable by using a ML approach, (h2) structured 127 versus minimalist upper effects on running kinematics and ground reaction forces are 128 classifiable by using a ML approach, and (h3) there are biomechanical changes due to shoe 
129 midsole resilience or upper structure that are subject-independent.

\section{Methods}

131 Subjects

Twenty-seven experienced non-professional male runners $(36.0 \pm 7.3$ years old,

strike pattern and with no experience in minimalist shoe participated in this study. All runners

had no major foot or ankle postural alterations or deformities, excessive static pronation or

2006); and did not present leg length discrepancy greater than $1 \mathrm{~cm}$. All athletes that participated in the Porto Alegre's Marathon in 2012 were invited by electronic media, and 158 athletes answered positively to participate in the study, however, only 27 effectively matched the eligibility criteria and came to the lab to be evaluated. Then a telephone call was made to select runners that fit to the inclusion criteria and scheduled the biomechanical evaluation in the laboratory. All subjects agreed to participate in the study approved by the Ethics Committee of the School of Medicine of the University of Sao Paulo (Ethical Application CEP-FMUSP: Protocol $\# 054 / 14)$ and signed a written consent form.

\section{Tested running shoes}

Four running shoes were especially developed by a local sportive shoe manufacturer.

The final masses of the 4 constructions were equivalent to avoid negative effects due to mass differences (Frederick 1984). All shoes were constructed using the same last, the same design 
151 of upper pieces, midsole geometry, and outsole. The hardness of cushioning materials was fixed

152 at 40 Asker C, measured by a durometer (GoTechAskerC, Taichung, Taiwan). The two shoe 153 uppers had the same design and shape, but the different structure and materials:

154 (1) SU - structured upper: $15 \mathrm{~mm}$ of soft foam in the heel collar and tongue, hard heel cup 155 involving the medial, lateral and posterior parts of the heel, synthetic pieces sewed in the vamp 156 and doubled fabric over the whole shoe (Figure 1A).

157 (2) MU - minimalist upper: light-weight mesh, tongue without foam, without heel cup, and 158 almost all pieces of the upper were connected by means of heat fusion (Figure 1B).

159 Both cushioning materials were made of ethylene-vinyl acetate (EVA); were inserted in 160 the same rearfoot area within the midsole; and had an oval shape of $10 \mathrm{~mm}$ thickness, $50 \mathrm{~mm}$ 161 width, and 70mm length. Resilience was assessed by vertical resiliometer (GoTech GT7042-V1, 162 Taichung, Taiwan). The midsoles were: (1) LR - low resilience - 5\% of resilience (+/- 3\%) (Figure 163 1C), and (2) HR - high resilience - 55\% of resilience (+/- 3\%) (Figure 1D).

\section{Figure 1}

The first tested condition (condition 1 - upper SU and cushioning material LR) was the same for all runners; the other three testing conditions were randomized for each subject using simple draw. The other three conditions were: condition 2 - upper SU and cushioning material HR, condition 3 - upper MU and cushioning material LR, and condition 4 - upper MU and cushioning material HR. The subjects were asked to lace their shoes tightly and comfortably, in the same way they typically lace during their running practice.

\section{Experimental protocol}


173 passive-reflexive markers (14 $\mathrm{mm}$ diameter) were fixed on both lower limbs (two anterior

174 superior iliac spines; two posterior iliac spines; two lateral epicondyles of the knees; two 175 markers over the lower lateral $1 / 3$ surface of the thighs; two lateral malleolus; two markers 176 over the lower $1 / 3$ of the shank; two second metatarsal heads; two posterior surface of 177 calcaneous at the same height above the plantar surface of the foot as the toes markers) 178 according to Plug'n Gait marker set (Kadaba et al. 1990). The two foot markers were fixed on 179 the shoes (second metatarsal heads and calcaneous) after deep palpation of bone 180 prominences. The laboratory coordinate system was established at one corner of one force 181 plate, and all initial calculations were based on this global coordinate system. In Nexus software 182 (Vicon Nexus 1.7, Oxford, UK), each data sample from each lower limb segment (foot, shank, and thigh) was modeled as a rigid body with a local coordinate system that coincided with anatomical axes. Translations and rotations of each segment were reported relative to neutral positions defined during the static standing trial.

The program calculates the joint angles by means of a decomposition matrix based on Cardan sequences and six degrees of freedom model. The decomposition matrix describes the relationship between two local coordinate systems, one for each segment between which the relative angle is determined. The joint kinematics was considered as the movement of the distal segment in relation to the proximal; e.g. for determining the knee angle, the thigh was the proximal segment and the shank the distal one. The movements occur around 3 different axes which describe two definition of movement each: flexion/extension, abduction/adduction, and internal/external rotation (Hamill et al. 2014). 
195 Watertown, USA) embedded in the center of a $25 \mathrm{~m}$ walkway. Acquisitions of kinematic and

196 force data were synchronized by a 64 multichannel Vicon MX Giganet Lab and A/D converter.

197 Running velocity was kept between 9.5 to $10.5 \mathrm{~km} / \mathrm{h}$ (mean $10.1 \pm 0.5 \mathrm{~km} / \mathrm{h}$ ), monitored

198 by 2 photoelectrical sensors (Tecsistel Speed View, Novo Hamburgo, Brazil). Ten trials per

199 subject for each shoe condition were collected, resulting in 40 trials on the dominant limb. The

200 limb dominance was defined as the leg used to kick a soccer ball (Greenberger \& Paterno 1995).

201

202

Biomechanical data analysis

203

In accordance with many studies in biomechanics (Muniz et al. 2010b; Nigg et al. 2012),

204

we adopted a Butterworth filter (implemented using the original code from MATLAB) to

minimize noise, and the marker coordinates were filtered using a $12 \mathrm{~Hz}$ zero-lag fourth-order

low-pass Butterworth filter. Force data was filtered with a $300 \mathrm{~Hz}$ zero-lag fourth-order low-

pass Butterworth filter also implemented in a MATLAB code. The angular and force data from initial contact to take-off were normalized in stance time (interpolated $0-100 \%$ ) and in magnitude by the body weight.

The 30 discrete kinematic features analyzed were: peak angles (degrees), angles at the

beginning of stance phase (degrees), instant of peak angle (seconds), range of motion from the beginning of stance phase to peak angle (degrees), and final angle of stance phase (degrees); for ankle, knee and hip joints; for sagittal and frontal planes of movement $(5 \times 3 \times 2)$. 
217 (body weight/ second), time of minimal vertical force in midstance (milliseconds), propulsion

218 rate (slope of curve between minimal vertical force in midstance and the 2VFP) (body weight/

219 second), minimum breaking antero-posterior force (body weight), breaking antero-posterior

220 impulse, median frequency of $\operatorname{VVFP}(\mathrm{Hz})$, time of stance phase (milliseconds), and decay rate

221 (slope of curve from 2VFP to the end of stance phase) (body weight/ second) were calculated.

222 The whole interpolated time-series of all three planes of motion (sagittal, frontal and

223 transversal) and forces (vertical, antero-posterior and medio-lateral) were also analyzed.

224 Usually, cross-sectional studies that investigate shoe effects in running biomechanics involve

225 high-dimensional and redundant datasets (Maurer et al. 2012; Nigg et al. 2012), and feature

226 selection techniques have been used to help identifying the biomechanical parameters that is

227 most influenced by shoe characteristics (Hoerzer et al. 2015; Maurer et al. 2012; Nigg et al.

228 2012). This was the main reason why we chose to include both discrete and whole time-series

229 points in the analysis. As explained in the next sections, we adopted an approach based on

230 ANN, which can receive large numbers of data simultaneously and the pieces of data do not

231 have to be isolated from each other (Barton \& Lees 1997).

232

233 Machine learning approach

234 To assess the effects of shoe interventions, many studies have generated high235 dimensional and redundant datasets (Maurer et al. 2012; Nigg et al. 2012), which impose 236 challenges for understanding an underlying phenomenon of interest (Guyon et al. 2006; Yu \& 237 Liu 2003). To overcome these challenges, Machine Learning (ML) techniques have been 238 adopted to find patterns on biomechanical data (Begg \& Kamruzzaman 2005; Hoerzer et al. 
239 2015; Maurer et al. 2012; Muniz et al. 2010b; Nigg et al. 2012). ML aims to learn from data. In a

240 typical classification scenario, we have a categorical outcome (like low vs high shoes resilience)

241 that we wish to predict or classify based on a set of features or variables (like ground reaction

242 forces). On the basis of a training set of data, we observe the outcome and feature

243 measurements for a set of instances (like the subject's trials) (Hastie et al. 2001). Using this

244 data, we build a classification model, which will enable us to classify the outcome for new

245 unseen instances. A good model is one that accurately classify such an outcome. This scenario

246 characterizes a supervised learning problem since each observed instance involves the outcome

247 variable (i.e., the desired output value) to guide the learning process.

248 A typical supervised classification approach consists of two parts: (i) variable or feature

249 selection and (ii) classification. Information Gain (IG) and Artificial Neural Networks (ANN) are

$250 \mathrm{ML}$ techniques that have been successfully used for feature selection and classification in many

251 areas. Whereas ANN have been used to classify patterns in biomechanics studies (Hastie et al.

252 2001; Muniz et al. 2010b; Rupérez et al. 2012; Witten et al. 2011), IG has not been explored in

253 biomechanics. Instead of IG, Principal Components Analysis (PCA) has been a popular technique

254 to select/construct features on walking and running biomechanical data (Hoerzer et al. 2015;

255 Maurer et al. 2012; Muniz et al. 2010b; Nigg et al. 2012). Support Vector Machine (SVM) and

256 Artificial Neural Networks (ANN) are techniques that have been used in classification tasks, like

257 in distinguishing effects of velocity (Joo et al. 2014), aging (Begg \& Kamruzzaman 2005; Fukuchi

258 et al. 2011; Wu et al. 2007), gender (Baltich et al. 2015; Maurer et al. 2012), diseases (Muniz et

259 al. 2010a; Muniz et al. 2010b; Muniz \& Nadal 2009; Nüesch et al. 2012) and footwear conditions

260 (Trudeau et al. 2015). As a result, age, gender (Maurer et al. 2012; Nigg et al. 2012) and the 
261 inter-subject's movement variability (Federolf et al. 2013; von Tscharner et al. 2013) are

262 intrinsic factors that have shown more influence than shoes characteristics on running 263 biomechanics.

264 Therefore, when the problem involves assessing the effects of shoes interventions, the

265 feature selection stage has to be carefully conducted since it plays a critical role in minimizing

266 bias and the influence of such intrinsic factors. In this context, even PCA has limitations (von

267 Tscharner et al. 2013), and a subject-independent analysis on shoes interventions is still an

268 open issue in the literature. In contrast to PCA, IG is a supervised method that ranks variables

269 individually without applying data transformations, and it has the potential to facilitate the

270 interpretation of the influence of a single variable on the underlying classification task (Begg \&

271 Kamruzzaman 2005; Muniz \& Nadal 2009).

272 As many studies in the literature (Joo et al. 2014; Oh et al. 2013; Schöllhorn 2004), we

273 adopted ANN for classification, even though SVM has been also successfully used for the same

274 task (Begg \& Kamruzzaman 2005; Maurer et al. 2012; Nigg et al. 2012; Trudeau et al. 2015).

275 Both SVM and ANN have been producing state-of-the-art results, even though some

276 comparative studies (Begg \& Kamruzzaman 2005; Fischer et al. 2011; Muniz et al. 2010b; Yang

277 et al. 2012) have indicated a slight advantage in favor of SVM. However, SVM were originally

278 designed for binary classification, which involves only two classes (e. g. low versus high

279 resilient), and an effective way of extend it for multiclass classification is a research issue (Hsu \&

280 Lin 2002). On the other hand, an ANN maps straightforwardly into multiclass classification

281 without requiring any further adjustments to approach the problem. In this study, although we

282 have approached shoes resilience and upper characteristics as two binary and independent 
283 classification problems, a natural extension of this study will focus on the fact that the effects

284 on running may be related to not only single shoes characteristics, but also to the combination

285 of them, resulting in a classification problem with more than 2 classes. In this context, ANN

286 represents a more stable scenario for further comparisons between binary and multiclass

287 classification results.

288

289 Input variables and feature selection by IG

The 3D joint angular displacement time series was vectorized to a 1254 dimensional

vector (3 lower limb joints x 3 planes of angular displacement x 101 interpolated data points +3

vectors of ground reaction forces $\times 101$ interpolated data points +42 discrete calculated kinetic and kinematic features). An input data matrix $M$ was then created (10 trials $\mathrm{x} 4$ shoe condition $\mathrm{x}$

27 subjects), resulting in a matrix dimension of $1080 \times 1254$. The 1080 lines of $M$ represented each subject trial in terms of 1254 input variables, some of which may be more affected by specific characteristics of shoe design. In this context, finding a small subset of input variables is redundant subset of features that would improve the results of the classification.

It was not practical to test all subsets of the 1254 input variables/columns available, then we used IG to rank the variables in a decreasing order of relevance. As a supervised method, IG ranks an input variable $X$ according to their discriminative power to separate the subject's trials in terms of a target variable $C$, like shoes resilience or upper. Usually, a distinct value $c_{i}$ of a target variable $C$ is known as a class. In our study, $C=\{l o w, h i g h\}$ for resilience and $C=$ \{minimalist,structured $\}$ for upper. IG is a correlation measure based on the 
305 information-theoretical concept of entropy, and the entropy of a variable $C$ is defined as in eq.

306 (1) (Yu \& Liu 2003):

307

$$
\mathrm{H}(\mathrm{C})=-\sum_{\mathrm{i}} \mathrm{P}\left(\mathrm{c}_{\mathrm{i}}\right) \log _{2}\left(\mathrm{P}\left(\mathrm{c}_{\mathrm{i}}\right)\right)
$$

308

309

310

311

312

313

315

316

317

318

319

320

321

322

323

324

325

326

and the entropy of $C$ after observing values of a variable $X$ is defined as eq. (2)

$$
H(C \mid X)=-\sum_{j} P\left(x_{j}\right) \sum_{i} P\left(c_{i} \mid x_{j}\right) \log _{2}\left(P\left(c_{i} \mid x_{j}\right)\right)
$$

where $P\left(c_{i}\right)$ is the prior probabilities for the values of $C$, and $P\left(c_{i} \mid x_{j}\right)$ is the posterior probabilities of $C$ given the values of $X$. The amount by which the entropy of $C$ decreases reflects additional information about $C$ provided by $X$, which is called information gain and it is given by eq. (3)

$$
\mathrm{IG}(\mathrm{C} \mid \mathrm{X})=\mathrm{H}(\mathrm{C})-\mathrm{H}(\mathrm{C} \mid \mathrm{X})
$$

In our experiments, diverse subsets of variables were tested; they contained an increasing number of variables $(25,50,100,150$ and 200) and bigger subsets were systematically formed by aggregating less relevant variables according to the IG criteria. This step was crucial to determine the smallest number of variables that achieved accurate discrimination of the resiliencies and upper structures. In this context, the greatest subset of variables involved in our experiments included 200 variables, since our analysis indicated that 200 variables were enough to evaluate the hypotheses of this study.

\section{Classification Procedure}

As described previously, lines in the matrix $M$ are instances of subject's trials, and each trial belongs to a class $c_{i}$ of resilience (low or high) and a class of upper (minimalist or structured). As a supervised classification approach, a subset of subject's trials was used for 
327 training and fitting a classification model, and the rest of the subject's trials were used for

328 validating the model. To estimate how accurately the classification model will perform in 329 practice, we adopted the standard k-fold-cross-validation, which divides the subject's trials into

$330 \mathrm{k}$ mutually exclusive folds of nearly equal size: $\mathrm{k}-1$ folds are used for training, and the remaining

331 fold for testing. The procedure repeats $k$ times, such that each fold is used once for validation.

332 For this reason, the validation results are usually averaged over the $\mathrm{k}$ rounds. For each training

333 round, a subset of variables was selected by using IG, their values were scaled from -1 to 1 , and

334 then an ANN learning model was trained.

335 The classification accuracy of the resulting ANN model was then computed on the test

336 fold. Because we are dealing with binary classification problems (i.e. two classes of resilience

337 and upper), accuracy is given in terms of the entries of a confusion matrix for positive and

338 negative classes (Han et al. 2011), as shown in Eq. (4). Given two classes (e.g. low and high

339 resilience), we can express in terms of positive trials (trials of one class, e.g. resilience $=$ low)

340 versus negative trials (e.g. resilience $=$ high). True positives $(T P)$ refer to the positive trials that

341 were correctly classified by the ANN model, while true negatives $(T N)$ are the negative trials

342 that were correctly classified by the ANN model. Similarly, false positives (FP) and false

343 negatives $(F N)$ are the negative and positive trials, respectively, that were incorrectly classified

344 by the ANN model.

$$
\text { Accuracy }=\frac{T P+T N}{T P+T N+F N+F P}
$$

347 (Fischer et al. 2011): an input, an output and a single hidden layer (Hastie et al. 2001). The 
348 number of entries in the input layer corresponds to the number of selected variables in the

349 feature selection stage, and the output layer consists of 2 neurons, which corresponds to each

350 class $c_{i}$ in our target variables, i.e. $C=\{$ low, high $\}$ for resilience or $C=$

$351\{$ minimalist,structured $\}$ for upper. As an ANN requires a parameter setting, which is still a

352 research issue, we perform an exhaustive searching through a subset of parameters values: the

353 number of neurons in the hidden layer was selected from the $\operatorname{set}\{10,25,50,75\}$; the learning

354 rate $\in\{0.05,0.15,0.25\}$ and the number of training cycles $\in\{300,700,1100,1500\}$. To

355 reduce the risk of overfitting, we adopted the decay procedure (Hastie et al. 2001), as

356 implemented in the RapidMiner software.

357 We conducted the classification in two contexts:

358 I. a 4-fold-cross-validation for each subject (40 trials) to assess the existence of effects from

359 shoes conditions, finding one accuracy value to discriminate the shoe condition for each subject

360 and for each subset of features. The purpose of considering trials of one subject only is to

361 conclude about our assumptions h1 and h2 in a context that avoids the influence of subject's

362 intrinsic factors.

363 II. a standard 10-fold-cross-validation involving all subjects' trials to assess the existence of 364 subject-independent changes induced by the shoes interventions, finding one accuracy value 365 for each subset of features.

By comparing classification accuracies between the contexts I and II, it was possible to

367 analyze the subject-dependency of the results and provisionally evaluate a pattern induced by

368 different resilience and upper conditions. Classification accuracy of higher than $80 \%$ was 369 considered good (Hoerzer et al. 2015), and we reported results when the ANN method 
370 achieved the best classification accuracy. The machine learning procedures were conducted in

371 the software RapidMiner (v.5.3.015, Dortmund, Germany).

372

373 Results

All 1254 variables were involved in the experiments of resiliencies and uppers. In both cases, the accuracy of discriminating the effect of shoe conditions indicated that context I outperformed context II.

The composition of the most relevant features subsets according to IG are detailed in

Tables 1 and 2 for the midsole resilience and upper structures comparisons, respectively.

Although forces have been the most discriminative variables for both midsole and upper, the top 5 features for upper and resilience came from different components, respectively vertical and medio-lateral forces.

\section{Table 1 and 2}

383

384

385

386

387

\section{Resilience effect}

In context II, 200 variables were sufficient to distinguish midsole resiliencies with an accuracy of $84.8 \%$ (Figure 2, red line). The accuracies indicated that context I, which considers only trials of a single subject, outperformed context II for all subsets of features (Figure 2, blue line). A mean accuracy of $89.4 \%( \pm 8.3 \%)$ to classify the two resiliencies was reached by considering only the 25 most relevant features, while the best accuracy of $93.9 \%( \pm 5.0)$ to classify the two resiliencies was reached with the 150 most relevant features.

Figure 2 

medio-lateral force, between $6 \%$ and $10 \%$ of stance phase.

\section{Figure 3}

Upper structure effect

The results indicated that the upper structures effects were less complex than the

cushioning materials ones. In the context II, accuracy higher than $85 \%$ was achieved by

considering only 25 variables to differentiate upper structures (Figure 4, red line). As in the case of resiliencies, results on uppers shown that context I outperformed context II; it was possible variables (Figure 4, blue line).

\section{Figure 4}

Among the 200 most relevant variables to discriminate structured and minimalist

uppers 11 of them came from the discrete biomechanical variables ( 9 force variables and 2

406 kinematic variables) (Figure 5). The 5 most relevant variables to discriminate between uppers

407 were vertical forces from $11 \%$ to $14 \%$ of stance phase and the first peak. 

results confirmed our first and second hypotheses because it was possible to observe the effects on running kinematics and kinetics caused by low and high resilient cushioning midsoles and structured and minimalist uppers by the adopted ML approach. IG was efficient in selecting important features, as was confirmed by the proportionally slower increase in classification accuracy with respect to increasing numbers of input features (Figure 4). The top biomechanical variables in the IG rank may be considered the most responsible for distinguishing the effects of the upper structures and midsole resiliencies.

When analyzing all subjects together, the methodology successfully differentiated the two resiliencies with $84.8 \%$ accuracy using 200 variables and the two shoe uppers with $85.3 \%$ accuracy using 25 variables, which is higher than the classification rate threshold of $80 \%$ chosen by Hoerzer et al. (2015). Intra-subject analysis increased the classification accuracy for resiliencies to a mean of $89.4 \%$ and for uppers using just 25 variables to a mean of $93.4 \%$. This indicated that the adopted $\mathrm{ML}$ analysis achieved more accuracy to identify different conditions (cushioning materials and shoe uppers) within a given subject than within the set of all subjects, which is consistent with the higher inter-subject variability. discriminating midsole resilience were mainly forces, ankle flexion-extension, and hip rotation variables. Sinclair et al. (2014) reported higher tibial acceleration, calcaneus eversion and internal rotation of tibia when running with high resilience midsole shoes. However, due to the 
432 part of them to other parts of the shoe (differences in the upper, for example). According to our

433 results, the main biomechanical alterations caused by different midsole resilience will be on

434 GRFs, sagittal plane of ankle and transversal plane of hip. Considering the discrete variables

435 that differentiated the resiliencies, 4 were related to vertical force (first peak and minimal

436 force) and 2 were related to ankle kinematics (dorsiflexion and eversion). Resilience causes

437 significant effects on the initial impact of the heel with the ground while running. The

438 cushioning materials were inserted only under the heel part of the shoe; considering we

439 focused our study on rearfoot strikers, it was acceptable that the resilience especially

440 influenced the variables related to this first part of the stance phase.

441 The 200 most relevant features that discriminated the two resiliencies were distributed

442 in short time windows spread over all cycle periods of the kinematic and kinetic time series.

443 This does not mean, however, that these 200 features were equally relevant for all 27 subjects.

444 These short and sparse windows corresponded to individual patterns of biomechanical

445 responses, or a group of individuals with the same biomechanical responses, which leads us to

446 refute part of the third hypothesis and conclude that changes due to shoe midsole resilience

447 seemed to be subject-dependent. This means that is possible to discriminate the two

448 resiliencies with a fewer number of variables and with a higher accuracy if we analyze

449 individualized biomechanical data. In summary, each runner responded differently to resilience

450 in the cushioning materials, changing a different pool of biomechanical variables that represent

451 distinct motor strategies. According to the "muscle tuning paradigm" proposed by Nigg and

452 Wakeling (2001), the individualized ability to modify the muscle tonus in response to impact

453 stimuli is one of the causes of different adaptabilities to shoes observed among subjects. 
454 Additionally, the most discriminative kinematic features were not found at the same time

455 periods as the most discriminative kinetic features in the stance phase, suggesting that

456 kinematics adjustments in the lower limbs caused by shoe changes might not be influencing

457 impact attenuation. This differs from what Hennig et al. (1996) and Milani et al. (1997)

458 suggested in their studies.

459 The upper structures classification had higher discriminative power than midsole

460 resiliencies, as was reflected in the higher accuracy levels of upper structure classification. The

46125 most relevant features for discriminating upper structures were composed mainly of force

462 variables (both discrete variables and time series features) and sagittal ankle variables. From

463 the 200 features, we found that the most relevant biomechanical variables for the classification

464 of uppers were concentrated in the first and last third of the stance phase for all three-force

465 components, sagittal plane of ankle, and for all planes of knee. The exceptions were for the

466 frontal and transverse planes of the ankle, which had the central third as the most relevant part

467 for classification. Therefore, changes in shoe uppers seem to be more subject-independent.

468 Eleven discrete biomechanical features were among the most relevant for classifying

469 upper structures. Nine were force variables related to first and second vertical peaks, minimal

470 vertical force, and breaking antero-posterior force; two were from ankle kinematics. According

471 to the relevant discrete parameters, it seemed that force features had highest discriminative

472 power, so were more relevant for differentiating upper structures than kinematic variables and

473 they were temporally distributed across the stance phase. The flexibility of the upper (due to

474 different applied materials) could affect the flexibility of the foot inside the shoe, its own

475 capability to absorb impact by stretching the foot arches and generate the propulsion force, so 
476 it is reasonable to assume that the force features across the entire stance phase are relevant

477 variables for distinguishing upper differences. The differences in structure probably affect 478 sensitivity to the ground, and consequently how runners modulate applied force to the ground. 479 As seen by Hagen and Hennig (2009), firmer foot contact with a shoe could result in lower 480 loading rates due to a better coupling between the foot and shoe, which in turn facilitates the 481 use of the impact-absorbing technology of the midsole.

In a training context, it would be ideal if each runner performed a biomechanical assessment of his or her running shoes. Our sample was constituted by rearfoot strikers, which were habituated to classic running shoes (not minimalist). This running strategy is the most prevalent among runners (de Almeida et al. 2015; Hasegawa et al. 2007) and according to Lieberman (2010), rearfoot strikers who grew up wearing shoes are more prone to be influenced by shoes conditions, so for this type of runners it is especially important to understand how this external factor (running shoe) changes (or not) each runner's particular running mechanics. Moreover, only few variables among the 1254 available (about 12\% for different cushioning materials and $4 \%$ for different shoe uppers) are evidently necessary to detect alterations of running biomechanics with high accuracy $(93.9 \% \pm 5.0 \%$ and $94.3 \% \pm 4.5 \%$, respectively). This result is plausible with the theory of "functional groups" (Hoerzer et al. 2015; Nigg et al. 2003) and it is appropriate to understand particular cases. Our results showed that runners have different responses to the materials used in the shoes and a general conclusion 495 arising from a heterogeneous sample may lead to wrong outcomes. Further studies could analyze the influence of smaller sub-groups with similar biomechanical responses to shoe 
498

499

500

501

502

503

504

505

506

507

508

509

510

511

512

513 518 subject-independent.

\section{Conclusion}

cushioning material affects the running mechanics for specific "types" of athletes. It was also possible to demonstrate that a biomechanical study of sports shoes isolating characteristics to be tested provided more specificity in the comprehension of the influence each part on running biomechanics. Future studies could explore other types of feature analysis from biomechanical data such as complexity (Sejdić et al. 2014), or sample entropy (Rathleff et al. 2010; Søndergaard et al. 2010) to distinguish running shoe properties and constructions from the perspective of signal variability.

The applied methodology based on feature selection by IG and classification by ANN successfully differentiated the high and low resilience materials and the structured and minimalist uppers with accuracies of higher than $85 \%$ using 200 features (16\% of 1254 available features). The classification of upper structures presented higher accuracy levels than that of midsole resilience probably due to higher inter-individual variability, but in both cases, the forces are among the 25 most relevant features subset. The ground reaction forces are the most important features to differentiate midsole resilience and the resilience caused valuable effects on initial heel impact while running. The different patterns of biomechanical response chosen by runners to adapt to different resilience probably led to lower accuracy levels for this classification. We can therefore conclude that biomechanical changes due to shoe midsole resilience seem to be subject-dependent and changes due to upper structure seem to be

519 
520

521

522

523

524

525

526

527

528

529

530

531

532

533

534

535

536

537

538

539

540

541

542

543

544

545

546

547

548

549

550

551

552

553

554

555

556

\section{Acknowledgements}

We would like to thank Dass Nordeste Calçados e Artigos Esportivos Inc. for supplying

the shoes prototypes and the laboratory used in this study, and Alexandro Rodrigo da Rosa and

Jonas Schneider for designing and developing the shoes used in this study.

\section{References}

Azevedo AP, Brandina K, Bianco R, Oliveira VH, Souza JR, Mezencio B, Amadio AC, and Serrão JC. 2012. Effects of replica running shoes upon external forces and muscle activity during running. J Sports Sci 30:929-935. 10.1080/02640414.2012.682080

Baltich J, Maurer C, and Nigg BM. 2015. Increased vertical impact forces and altered running mechanics with softer midsole shoes. PLoS One 10:e0125196. 10.1371/journal.pone.0125196

Barton JG, and Lees A. 1997. An application of neural networks for distinguishing gait patterns on the basis of hip-knee joint angle diagrams. Gait \& Posture 5:28-33. http://dx.doi.org/10.1016/S09666362(96)01070-3

Begg R, and Kamruzzaman J. 2005. A machine learning approach for automated recognition of movement patterns using basic, kinetic and kinematic gait data. J Biomech 38:401-408. 10.1016/j.jbiomech.2004.05.002

Braunstein B, Arampatzis A, Eysel P, and Brüggemann GP. 2010. Footwear affects the gearing at the ankle and knee joints during running. J Biomech 43:2120-2125. 10.1016/j.jbiomech.2010.04.001

Cheung RT, Wong MY, and Ng GY. 2011. Effects of motion control footwear on running: a systematic review. J Sports Sci 29:1311-1319. 10.1080/02640414.2011.591420

Clarke TE, Frederick EC, and Cooper LB. 1983. Effects of shoe cushioning upon ground reaction forces in running. Int J Sports Med 4:247-251. 10.1055/s-2008-1026043

de Almeida MO, Saragiotto BT, Yamato TP, and Lopes AD. 2015. Is the rearfoot pattern the most frequently foot strike pattern among recreational shod distance runners? Phys Ther Sport 16:2933. 10.1016/j.ptsp.2014.02.005

Dixon SJ. 2008. Use of pressure insoles to compare in-shoe loading for modern running shoes. Ergonomics 51:1503-1514. 10.1080/00140130802239562

Federolf PA, Boyer KA, and Andriacchi TP. 2013. Application of principal component analysis in clinical gait research: identification of systematic differences between healthy and medial kneeosteoarthritic gait. J Biomech 46:2173-2178. 10.1016/j.jbiomech.2013.06.032

Fischer A, Do M, Stein T, Asfour T, Dillmann R, and Schwameder H. 2011. Recognition of individual kinematic patterns during walking and running - A comparison of artificial neural networks and support vector machines. International Journal of Computer Science in Sport 10:63-67.

Frederick EC. 1984. Physiological and ergonomics factors in running shoe design. Appl Ergon 15:281-287.

Fukuchi RK, Eskofier BM, Duarte M, and Ferber R. 2011. Support vector machines for detecting agerelated changes in running kinematics. J Biomech 44:540-542. 10.1016/j.jbiomech.2010.09.031 
557

Greenberger HB, and Paterno MV. 1995. Relationship of knee extensor strength and hopping test performance in the assessment of lower extremity function. J Orthop Sports Phys Ther 22:202206. 10.2519/jospt.1995.22.5.202

Guyon I, Gunn S, Nikravesh M, and Zadeh LA. 2006. Feature extraction: Foundations and applications. New York: Springer-Verlag Berlin Heidelberg.

Hagen M, and Hennig EM. 2009. Effects of different shoe-lacing patterns on the biomechanics of running shoes. J Sports Sci 27:267-275. 10.1080/02640410802482425

Hamill J, Selbie WS, and Kepple TM. 2014. Three-Dimensional Kinematics, Research Methods in Biomechanics. Human Kinetics. 2nd ed ed.

Han J, Pei J, and Kamber M. 2011. Data mining: concepts and techniques.: Elsevier.

Hardin EC, van den Bogert AJ, and Hamill J. 2004. Kinematic adaptations during running: effects of footwear, surface, and duration. Med Sci Sports Exerc 36:838-844.

Hasegawa H, Yamauchi T, and Kraemer WJ. 2007. Foot strike patterns of runners at the $15-\mathrm{km}$ point during an elite-level half marathon. J Strength Cond Res 21:888-893. 10.1519/R-22096.1

Hastie T, Tibshirani R, and Friedman J. 2001. The Elements of Statistical Learning. New York, NY, USA: Springer New York Inc.

Hennig EM. 2011. Eighteen years of running shoe testing in Germany - a series of biomechanical studies Footwear Science 3:71-81.

Hennig EM, Valiant GA, and Liu Q. 1996. Biomechanical variables and the perception of cushioning for running in various types of footwear. Journal of Applied Biomechanics 12:143 - 150.

Hoerzer S, von Tscharner V, Jacob C, and Nigg BM. 2015. Defining functional groups based on running kinematics using Self-Organizing Maps and Support Vector Machines. J Biomech 48:2072-2079. 10.1016/j.jbiomech.2015.03.017

Hsu CW, and Lin CJ. 2002. A comparison of methods for multiclass support vector machines. IEEE Trans Neural Netw 13:415-425. 10.1109/72.991427

Joo S, Oh SE, Sim T, Kim H, Choi CH, and Koo H. 2014. Prediction of gait speed from plantar pressure using artificial neural networks. Expert Systems with Applications 41:7398 - 7405.

Kadaba MP, Ramakrishnan HK, and Wootten ME. 1990. Measurement of lower extremity kinematics during level walking. J Orthop Res 8:383-392. 10.1002/jor.1100080310

Kersting UG, and Brüggemann GP. 2006. Midsole material-related force control during heel-toe running. Res Sports Med 14:1-17. 10.1080/15438620500528158

Lieberman DE, Venkadesan M, Werbel WA, Daoud AI, D'Andrea S, Davis IS, Mang'eni RO, and Pitsiladis Y. 2010. Foot strike patterns and collision forces in habitually barefoot versus shod runners. Nature 463:531-535. 10.1038/nature08723

Macera CA, Pate RR, Powell KE, Jackson KL, Kendrick JS, and Craven TE. 1989. Predicting lower-extremity injuries among habitual runners. Arch Intern Med 149:2565-2568.

Maclean CL, Davis IS, and Hamill J. 2009. Influence of running shoe midsole composition and custom foot orthotic intervention on lower extremity dynamics during running. J Appl Biomech 25:5463.

Maurer C, Federolf P, von Tscharner V, Stirling L, and Nigg BM. 2012. Discrimination of gender-, speed-, and shoe-dependent movement patterns in runners using full-body kinematics. Gait Posture 36:40-45. 10.1016/j.gaitpost.2011.12.023

McNair PJ, and Marshall RN. 1994. Kinematic and kinetic parameters associated with running in different shoes. Br J Sports Med 28:256-260.

Milani TL, Hennig EM, and Lafortune MA. 1997. Perceptual and biomechanical variables for running in identical shoe constructions with varying midsole hardness. Clin Biomech (Bristol, Avon) 12:294300. 
604

605

606

607

608

609

610

611

612

613

614

615

616

617

618

619

620

621

622

623

624

625

626

627

628

629

630

631

632

633

634

635

636

637

638

639

640

641

642

643

644

645

646

647

648

649

Muniz AM, Liu H, Lyons KE, Pahwa R, Liu W, and Nadal J. 2010a. Quantitative evaluation of the effects of subthalamic stimulation on gait in Parkinson's disease patients using principal component analysis. Int J Neurosci 120:609-616. 10.3109/00207454.2010.504904

Muniz AM, Liu H, Lyons KE, Pahwa R, Liu W, Nobre FF, and Nadal J. 2010b. Comparison among probabilistic neural network, support vector machine and logistic regression for evaluating the effect of subthalamic stimulation in Parkinson disease on ground reaction force during gait. $J$ Biomech 43:720-726. 10.1016/j.jbiomech.2009.10.018

Muniz AM, and Nadal J. 2009. Application of principal component analysis in vertical ground reaction force to discriminate normal and abnormal gait. Gait Posture 29:31-35.

10.1016/j.gaitpost.2008.05.015

Nigg BM. 2010. Biomechanics of sport shoes. Alberta, Canada: Topline Printing Inc.

Nigg BM, Bahlsen HA, Luethi SM, and Stokes S. 1987. The influence of running velocity and midsole hardness on external impact forces in heel-toe running. J Biomech 20:951-959.

Nigg BM, Baltich J, Hoerzer S, and Enders H. 2015. Running shoes and running injuries: mythbusting and a proposal for two new paradigms: 'preferred movement path' and 'comfort filter'. Br J Sports Med. 10.1136/bjsports-2015-095054

Nigg BM, Baltich J, Maurer C, and Federolf P. 2012. Shoe midsole hardness, sex and age effects on lower extremity kinematics during running. J Biomech 45:1692-1697. 10.1016/j.jbiomech.2012.03.027

Nigg BM, Stefanyshyn D, Cole G, Stergiou P, and Miller J. 2003. The effect of material characteristics of shoe soles on muscle activation and energy aspects during running. J Biomech 36:569-575.

Nigg BM, and Wakeling JM. 2001. Impact forces and muscle tuning: a new paradigm. Exerc Sport Sci Rev 29:37-41.

Nüesch C, Valderrabano V, Huber C, von Tscharner V, and Pagenstert G. 2012. Gait patterns of asymmetric ankle osteoarthritis patients. Clin Biomech (Bristol, Avon) 27:613-618. 10.1016/j.clinbiomech.2011.12.016

Oh SE, Choi A, and Mun JH. 2013. Prediction of ground reaction forces during gait based on kinematics and a neural network model. J Biomech 46:2372-2380. 10.1016/j.jbiomech.2013.07.036

Rathleff MS, Olesen CG, Moelgaard CM, Jensen K, Madeleine P, and Olesen JL. 2010. Non-linear analysis of the structure of variability in midfoot kinematics. Gait Posture 31:385-390. 10.1016/j.gaitpost.2010.01.008

Redmond AC, Crosbie J, and Ouvrier RA. 2006. Development and validation of a novel rating system for scoring standing foot posture: the Foot Posture Index. Clin Biomech (Bristol, Avon) 21:89-98. 10.1016/j.clinbiomech.2005.08.002

Rupérez MJ, Martín-Guerrero JD, Monserrat C, and Alcañiz M. 2012. Artificial neural networks for predicting dorsal pressures on the foot surface while walking. Expert Systems with Applications 39:5349 - 5357. 10.1016/j.eswa.2011.11.050

Schöllhorn WI. 2004. Applications of artificial neural nets in clinical biomechanics. Clin Biomech (Bristol, Avon) 19:876-898. 10.1016/j.clinbiomech.2004.04.005

Schöllhorn WI, Nigg BM, Stefanyshyn DJ, and Liu W. 2002. Identification of individual walking patterns using time discrete and time continuous data sets. Gait Posture 15:180-186.

Sejdić E, Lowry KA, Bellanca J, Redfern MS, and Brach JS. 2014. A comprehensive assessment of gait accelerometry signals in time, frequency and time-frequency domains. IEEE Trans Neural Syst Rehabil Eng 22:603-612. 10.1109/TNSRE.2013.2265887

Sinclair J, Franks C, Fau-Goodwin J, Naemi R, and Chockalingam N. 2014. Influence of footwear designed to boost energy return on the kinetics and kinematics of running compared to conventional running shoes. Comparative Exercise Physiology 10:199-206. 
650

651

652

653

654

655

656

657

658

659

660

661

662

663

664

665

666

667

668

669

670

671

672

673

674

675

676

677

678

679

Sinclair J, Mcgrath R, Brook O, Taylor PJ, and Dillon S. 2016. Influence of footwear designed to boost energy return on running economy in comparison to a conventional running shoe. J Sports Sci 34:1094-1098. 10.1080/02640414.2015.1088961

Sun PC, Wei HW, Chen CH, Wu CH, Kao HC, and Cheng CK. 2008. Effects of varying material properties on the load deformation characteristics of heel cushions. Med Eng Phys 30:687-692. 10.1016/j.medengphy.2007.07.010

Søndergaard KH, Olesen CG, Søndergaard EK, de Zee M, and Madeleine P. 2010. The variability and complexity of sitting postural control are associated with discomfort. J Biomech 43:1997-2001. 10.1016/j.jbiomech.2010.03.009

Trudeau MB, von Tscharner V, Vienneau J, Hoerzer S, and Nigg BM. 2015. Assessing Footwear Effects from Principal Features of Plantar Loading during Running. Med Sci Sports Exerc. 10.1249/MSS.0000000000000615

Verdejo R, and Mills NJ. 2004. Heel-shoe interactions and the durability of EVA foam running-shoe midsoles. J Biomech 37:1379-1386. 10.1016/j.jbiomech.2003.12.022

von Tscharner V, Enders H, and Maurer C. 2013. Subspace identification and classification of healthy human gait. PLoS One 8:e65063. 10.1371/journal.pone.0065063

Wang L, Hong Y, and Li JX. 2012. Durability of running shoes with ethylene vinyl acetate or polyurethane midsoles. J Sports Sci 30:1787-1792. 10.1080/02640414.2012.723819

Witten IH, Frank E, and Hall MA. 2011. Data Mining: Practical machine learning tools and techniques. San Diego, CA, USA: Morgan Kaufmann.

Worobets J, Wannop JW, Tomaras E, and Stefanyshyn D. 2014. Softer and more resilient running shoe cushioning properties enhance running economy. Footwear Science 6:147-153.

Wu J, Wang J, and Liu L. 2007. Feature extraction via KPCA for classification of gait patterns. Hum Mov Sci 26:393-411. 10.1016/j.humov.2007.01.015

Yang M, Zheng H, Wang H, McClean S, Hall J, and Harris N. 2012. A machine learning approach to assessing gait patterns for Complex Regional Pain Syndrome Medical Engineering \& Physics 34:740 - 746.

Yu L, and Liu H. 2003. Feature Selection for High-Dimensional Data: A Fast Correlation-Based Filter Solution. Twentieth International Conference on Machine Learning. Washington DC. p 856-863. 


\section{Table $\mathbf{1}$ (on next page)}

IG rank subset for midsole resilience

Number of variables of each feature subset according to IG rank for midsole resilience comparison. 
1 Table 1 - Number of variables of each feature subset according to IG rank for midsole resilience 2 comparison.

\begin{tabular}{|c|c|c|c|c|c|}
\hline $\begin{array}{l}\text { RESILIENCE } \\
\text { COMPARISON }\end{array}$ & $\begin{array}{l}25 \text { most } \\
\text { relevant } \\
\text { variables }\end{array}$ & $\begin{array}{l}50 \text { most } \\
\text { relevant } \\
\text { variables }\end{array}$ & $\begin{array}{l}100 \text { most } \\
\text { relevant } \\
\text { variables }\end{array}$ & $\begin{array}{l}150 \text { most } \\
\text { relevant } \\
\text { variables }\end{array}$ & $\begin{array}{l}200 \text { most } \\
\text { relevant } \\
\text { variables }\end{array}$ \\
\hline Medio-lateral GRF & 9 & 11 & 14 & 17 & 18 \\
\hline Sagittal Ankle & 6 & 9 & 16 & 18 & 25 \\
\hline Transversal Hip & 3 & 12 & 17 & 33 & 39 \\
\hline Vertical GRF & 2 & 4 & 6 & 12 & 16 \\
\hline Antero-posterior GRF & 2 & 2 & 7 & 11 & 17 \\
\hline Discrete Force Variables & 2 & 3 & 4 & 4 & 4 \\
\hline Discrete Kinematic & 1 & 1 & 1 & 2 & 2 \\
\hline \multicolumn{6}{|l|}{ Variables } \\
\hline Frontal Knee & & 7 & 12 & 12 & 14 \\
\hline Sagittal Hip & & 1 & 19 & 35 & 38 \\
\hline Transversal Knee & & & 4 & 5 & 12 \\
\hline Frontal Ankle & & & & 1 & 5 \\
\hline Frontal Hip & & & & & 6 \\
\hline Transversal Ankle & & & & & 4 \\
\hline $\begin{array}{l}\text { \% of total features } \\
\text { (1254) }\end{array}$ & $2 \%$ & $4 \%$ & $8 \%$ & $12 \%$ & $16 \%$ \\
\hline
\end{tabular}




\section{Table 2 (on next page)}

IG rank subset for upper structure

Number of variables of each feature subset according to IG rank for upper structures comparison. 
1 Table 2 - Number of variables of each feature subset according to IG rank for upper structures

2 comparison.

\begin{tabular}{|c|c|c|c|c|c|}
\hline $\begin{array}{l}\text { UPPER STRUCTURE } \\
\text { COMPARISON }\end{array}$ & $\begin{array}{l}25 \text { most } \\
\text { relevant } \\
\text { variables }\end{array}$ & $\begin{array}{l}50 \text { most } \\
\text { relevant } \\
\text { variables }\end{array}$ & $\begin{array}{l}100 \text { most } \\
\text { relevant } \\
\text { variables }\end{array}$ & $\begin{array}{l}150 \text { most } \\
\text { relevant } \\
\text { variables }\end{array}$ & $\begin{array}{l}200 \text { most } \\
\text { relevant } \\
\text { variables }\end{array}$ \\
\hline Antero-posterior GRF & 10 & 19 & 33 & 38 & 40 \\
\hline Vertical GRF & 8 & 12 & 16 & 19 & 21 \\
\hline Discrete Force Variables & 4 & 4 & 4 & 6 & 9 \\
\hline Sagittal Ankle & 2 & 10 & 20 & 22 & 28 \\
\hline Medio-lateral GRF & 1 & 4 & 12 & 18 & 22 \\
\hline Transversal Knee & & 1 & 7 & 19 & 25 \\
\hline Frontal Ankle & & & 5 & 13 & 26 \\
\hline Transversal Ankle & & & 2 & 9 & 10 \\
\hline Discrete Kinematic & & & 1 & 2 & 2 \\
\hline \multicolumn{6}{|l|}{ Variables } \\
\hline Sagittal Knee & & & & 4 & 7 \\
\hline Transversal Hip & & & & & 6 \\
\hline Frontal Knee & & & & & 4 \\
\hline $\begin{array}{l}\% \text { of total features } \\
(1254)\end{array}$ & $2 \%$ & $4 \%$ & $8 \%$ & $12 \%$ & $16 \%$ \\
\hline
\end{tabular}

3 


\section{Figure 1}

Image of shoes prototypes used in the experiment

Illustration of testing shoes. (A) Structured Upper condition (SU). (B) Minimalist Upper condition (MU). (C) Low Resilience cushioning material condition (LR). (D) High Resilience cushioning material condition (HR).
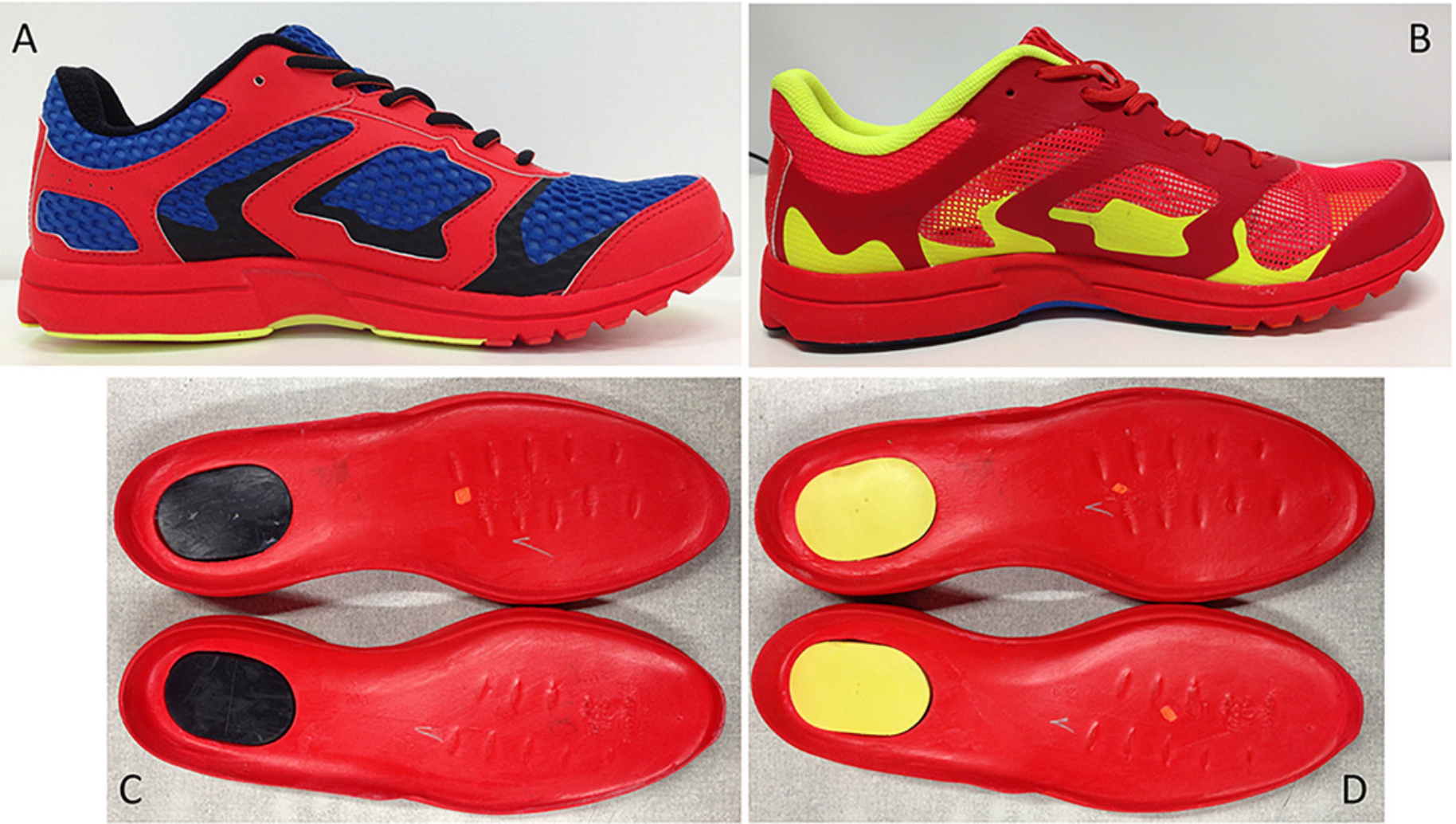
Figure 2

Accuracy levels to discriminate midsole resilience materials in various contexts considering different subsets of variables

Mean accuracy and standard deviation for each subset of input variables with the highest IG values to discriminate resilience materials. Red line represents the context II and considers all subjects together. Blue line represents the context I and considers each subject in isolation.

\section{$105 \%$}

$100 \%$

$95 \%$

$90 \%$

$85 \%$

$80 \%$

$75 \%$

$70 \%$

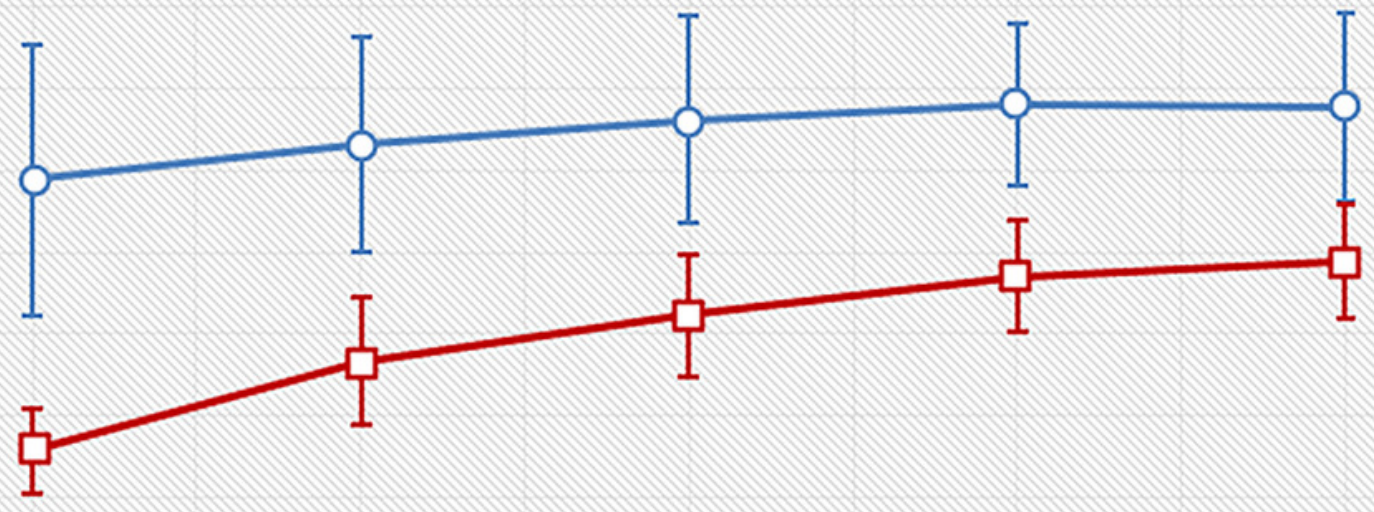

$65 \%$

$60 \%$

25

50

100

150

200

Number of variables with higher relevance according to IG 


\section{Figure 3}

Ground reaction force and Kinematics time-series during running with different resilience midsoles.

(A) Mean time series of ground reaction force for different resilience of cushioning materials.

(B) Mean time series of joints kinematics in all planes of motion for different resilience materials. Blue lines represent the low resilience cushioning condition and red dotted lines represent the high resilience cushioning condition. The 200 highest IG variables are highlighted in the yellow boxes. 
A
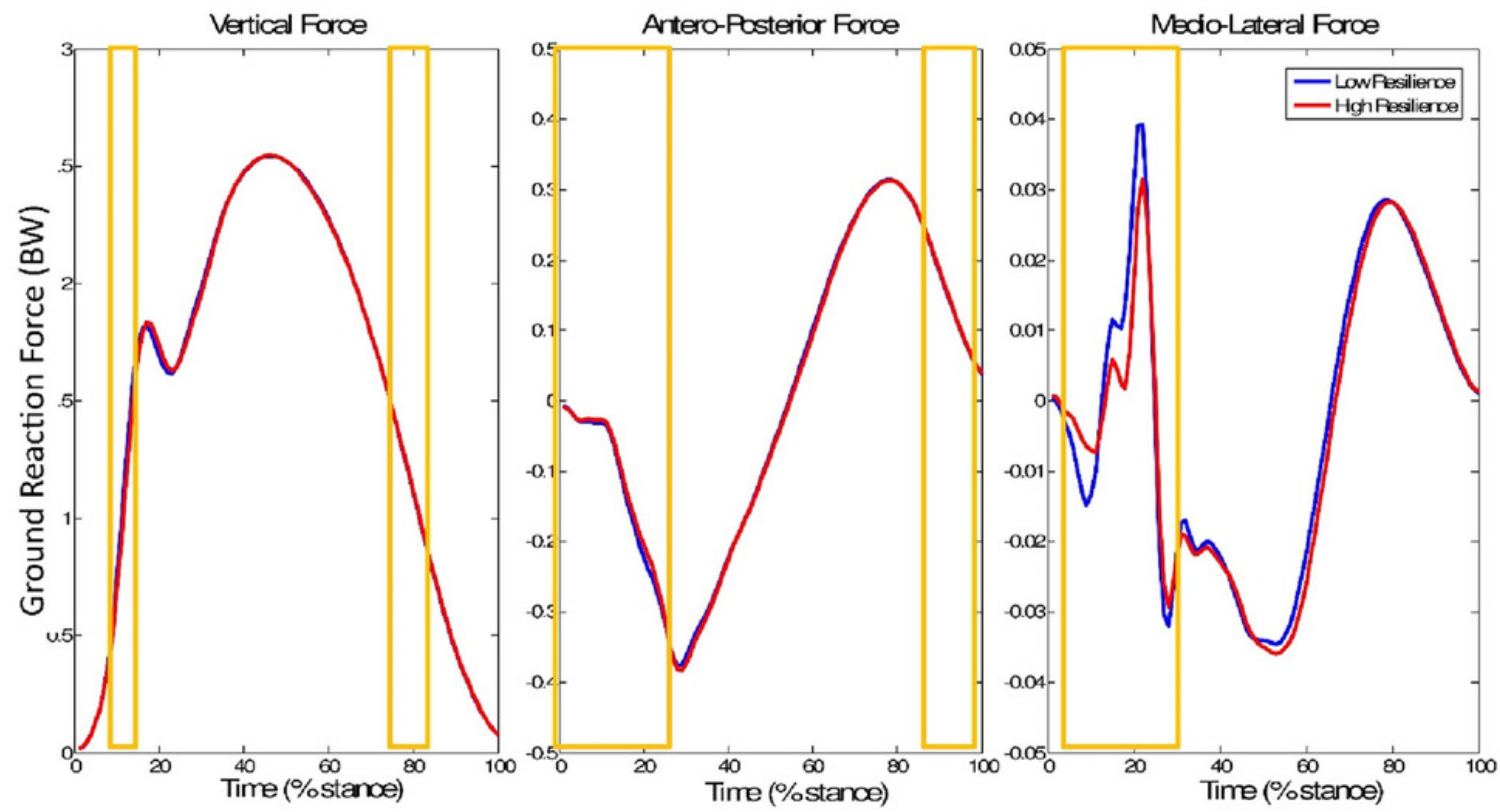

B
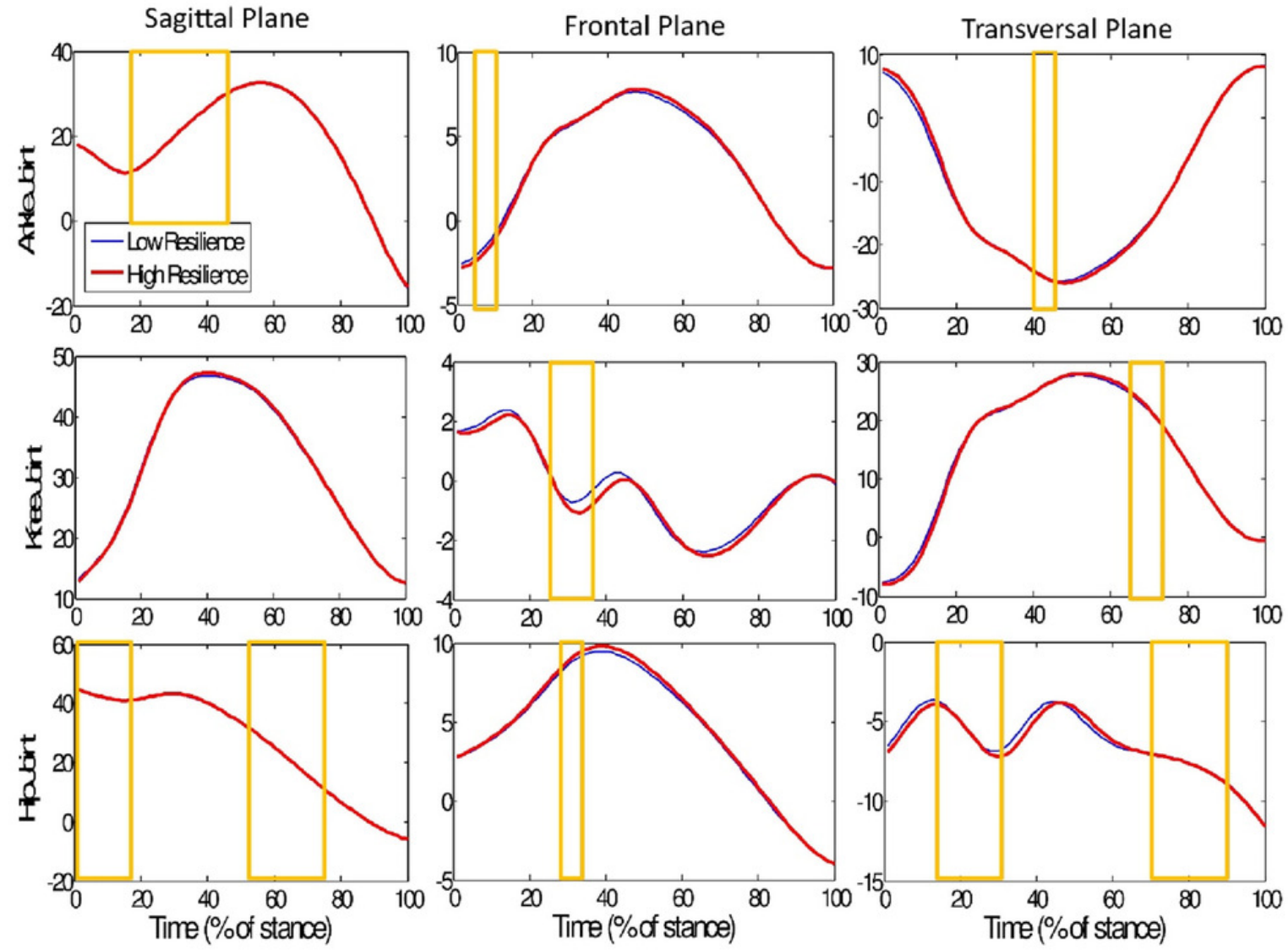

PeerJ reviewing PDF | (2016:10:13746:1:1:CHECK 14 Jan 2017) 
Figure 4

Accuracy levels to discriminate upper strustures in various contexts considering different subsets of variables

Mean accuracy and standard deviation for each subset of input variables with the highest IG values to classify upper structures. Red line represents the context II and considers all subjects together. Blue line represents the context I and considers each subject in isolation.

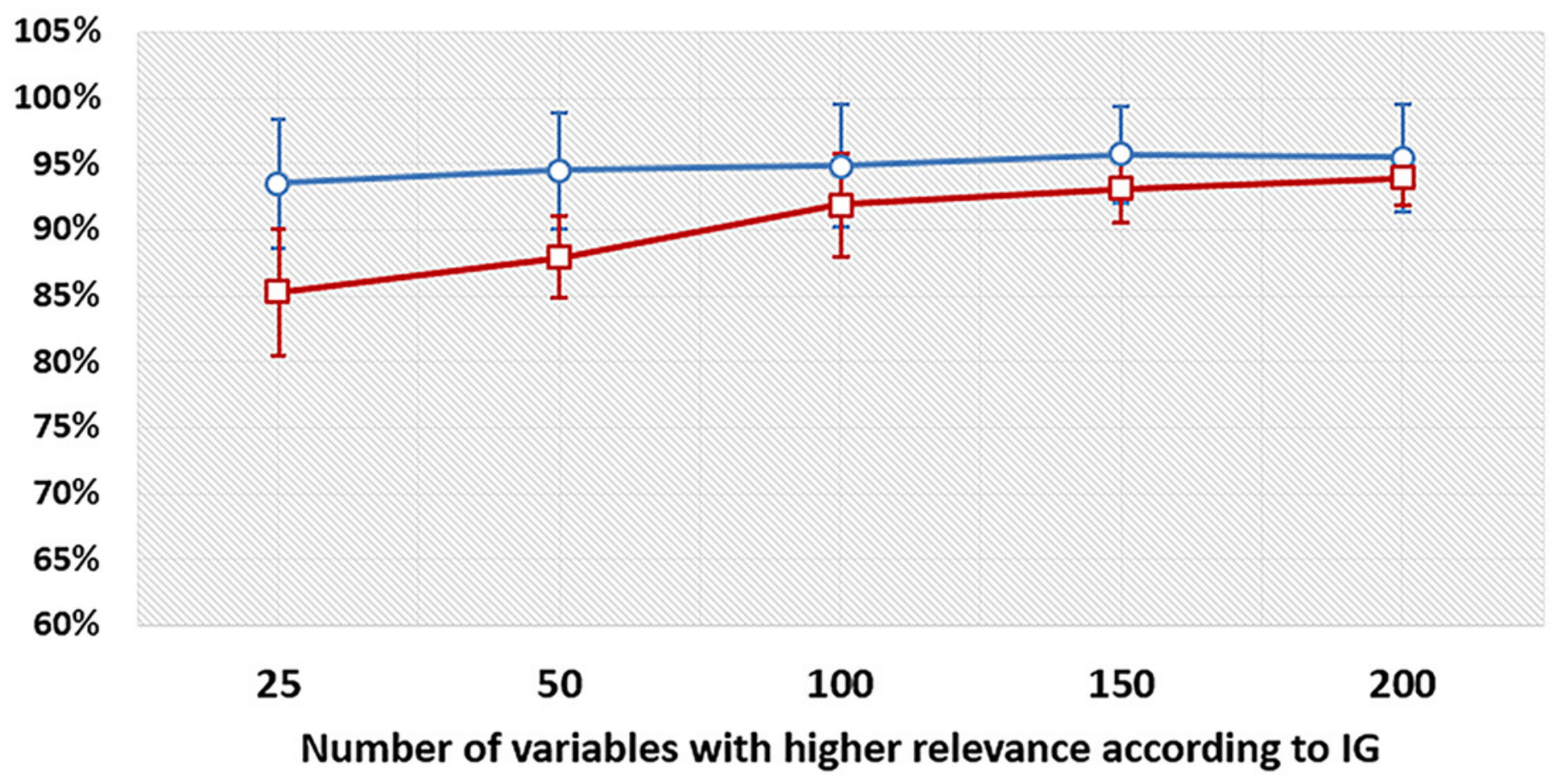




\section{Figure 5}

Ground reaction force and kinematics time-series during running with different shoe upper structures.

(A) Mean time series of ground reaction force for different shoe upper structures. (B) Mean time series of joints kinematics in all planes of motion for different shoe upper structures. Black lines represent the structured upper condition and Pink dotted lines represent the minimalist upper condition. The 200 highest IG variables are highlighted in the yellow boxes. 
A
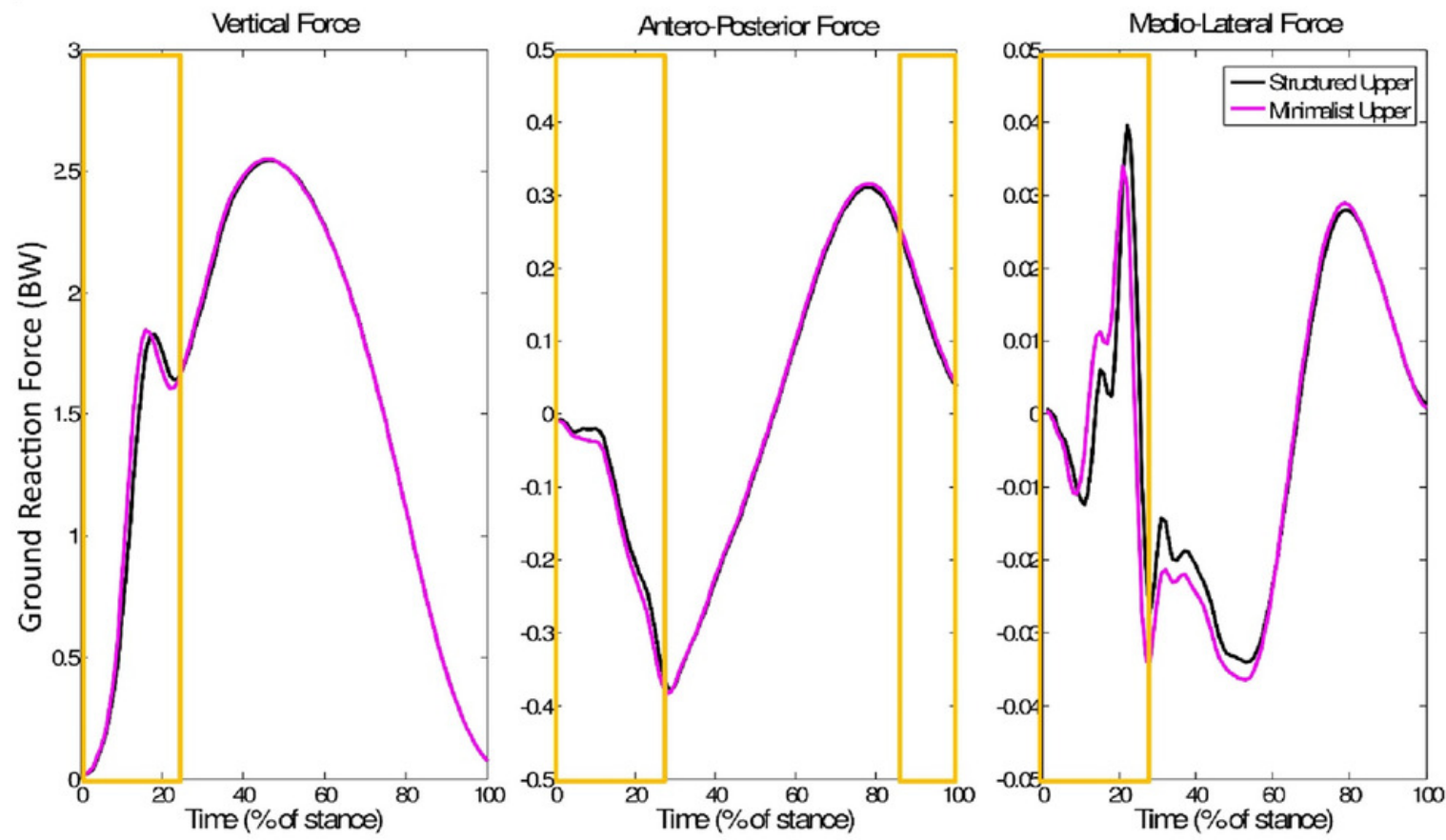

B
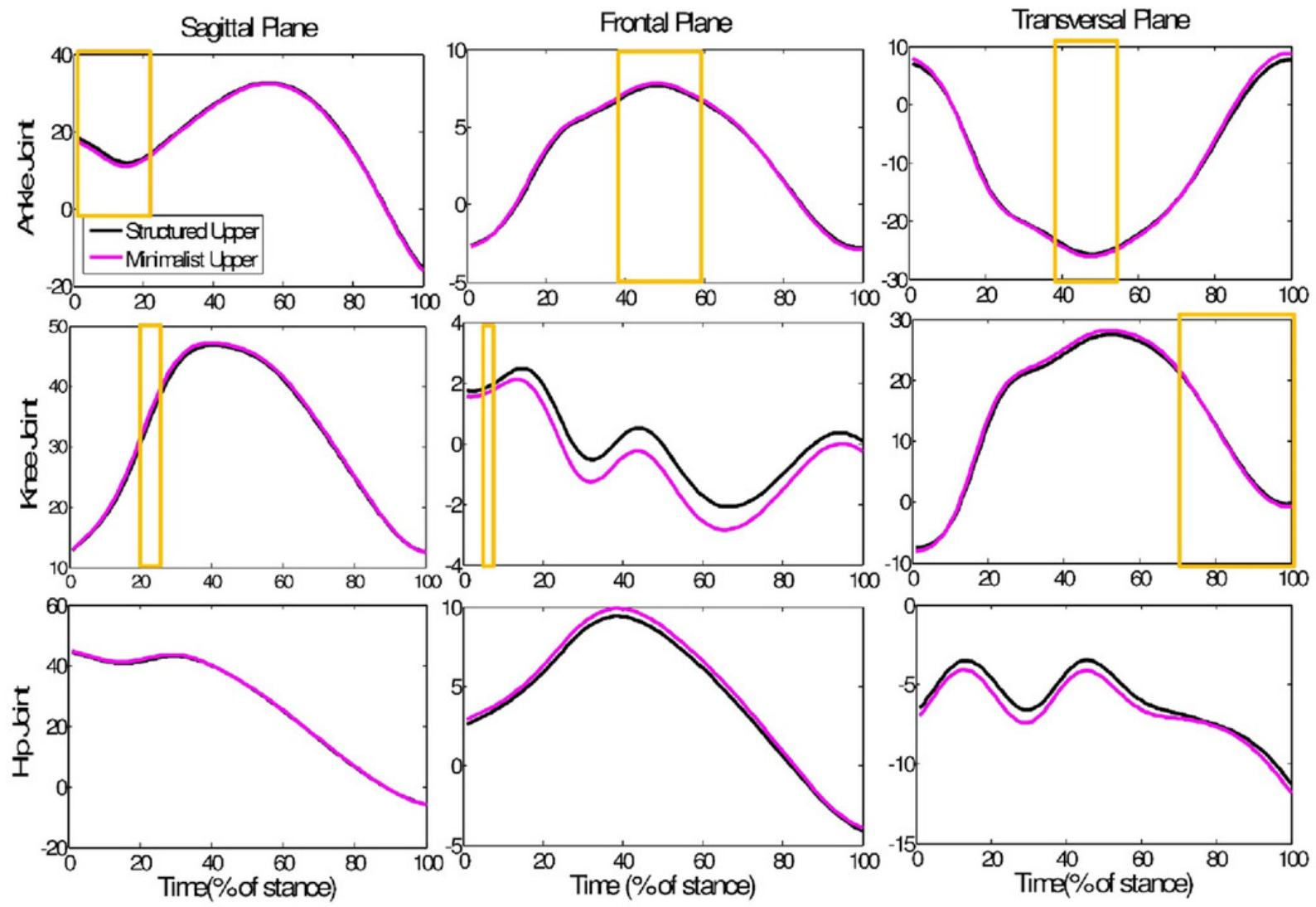

PeerJ reviewing PDF | (2016:10:13746:1:1:CHECK 14 Jan 2017) 